\title{
Surface-plasmon polaritons in a lattice of metal cylinders
}

\author{
J. M. Pitarke, ${ }^{1,2}$ J. E. Inglesfield, ${ }^{3}$ and N. Giannakis ${ }^{4}$ \\ ${ }^{1}$ CIC nanoGUNE Consolider, Mikeletegi Pasealekua 56, E-20009 Donostia, Basque Country, Spain \\ ${ }^{2}$ Materia Kondentsatuaren Fisika Saila, UPV/EHU, and Unidad Física Materiales CSIC-UPV/EHU, 644 Posta kutxatila, \\ E-48080 Bilbo, Basque Country, Spain \\ ${ }^{3}$ School of Physics and Astronomy, Cardiff University, Cardiff, CF24 3AA, United Kingdom \\ ${ }^{4}$ Department of Electronic Engineering, Queen Mary, University of London, London, E1 4NS, United Kingdom
}

(Received 9 November 2006; revised manuscript received 26 December 2006; published 24 April 2007)

Plasmon modes of a two-dimensional lattice of long conducting circular wires are investigated by using an embedding technique to solve Maxwell's equations rigorously. The frequency-dependent density of states is calculated for various values of the wave vector and the filling fraction. At low filling fractions, collective modes are all found to accumulate at the surface-plasmon frequency $\omega_{p} / \sqrt{2}, \omega_{p}$ being the bulk plasmon frequency. As the filling fraction increases, the interference between the electromagnetic fields generated by localized surface-plasmon polaritons leads to the presence of new resonances, whose frequency strongly depends on the interparticle separation. For touching wires, a number of multipole resonances fill the spectral range between dipole resonances, as occurs in the case of a three-dimensional packing of metal spheres.

DOI: 10.1103/PhysRevB.75.165415

PACS number(s): 73.22.Lp, 42.70.Qs, 42.25.Bs

\section{INTRODUCTION}

Over the last few years, a great deal of attention has been devoted to the investigation of metallodielectric photonic crystals, partially stimulated by the appearance of remarkable optical properties associated with the excitation of surfaceplasmon polaritons. ${ }^{1-6}$ These are self-sustained collective excitations corresponding to fluctuations of the surface electron density. ${ }^{7,8}$

To understand the properties of surface-plasmon polaritons, and their possible role in the optical properties of artificially structured metallodielectric metamaterials, we need the photonic band structure of lattices of metallic inclusions with frequency-dependent dielectric functions. Lattices of metallic rods, ${ }^{9}$ cylinders,${ }^{10-13}$ and spheres ${ }^{14-17}$ have all been studied up to now. Three-dimensional (3D) face-centered cubic lattices composed of metal spheres, their plasmon modes, and interaction with light were studied by Yannopapas et $a l .,{ }^{16}$ in the low filling fraction regime, and wide bands of multipole resonances were later found to occur as the filling fraction increases. ${ }^{17}$ Flat bands of localized surface plasmons were also found to occur in a two-dimensional periodic arrangement of long metallic cylinders. ${ }^{12}$ The impact of localized multipole plasmons on the optical absorption and the energy loss of 2D and 3D metallodielectric crystals was addressed in Refs. 18-20, by looking at the imaginary part of the so-called effective dielectric function of the composite.

In this paper, we consider the evolution of plasmon bands in a 2D square lattice made up of long metallic cylinders embedded in a homogeneous medium, as the cylinder size increases from the noninteracting limit (small radius compared to the lattice constant) to touching cylinders where interactions play a key role. We shall consider electromagnetic modes with the wave vector perpendicular to the cylinders, in which case the modes fall into two categories: $E$ (or $s$ ) polarization, in which the electric field is parallel to the cylinder axis, and $H$ (or $p$ ) polarization, in which the electric field lies in the 2D plane. We shall focus our attention on the plasmonic regime, where the radius of the cylinders is much smaller than the wavelength of the interacting electromagnetic radiation.

At long wavelengths, the behavior for $E$ polarization is the same as that of a homogeneous metal with a reduced plasmon frequency given by $\sqrt{f} \omega_{p},{ }^{10,18}$ where $f$ is the filling fraction and $\omega_{p}$ the bulk plasmon frequency of the metal cylinders. Conversely, for $H$ polarization surface plasmons can be excited, and due to the interference between their electromagnetic fields their frequencies vary strongly with the cylinder separation, which suggests that their frequency can be tuned by changing the separation. Here we shall show that for this polarization and in the limit of touching spheres multipole resonances fill the spectral range between dipole resonances, as occurs in the case of 3D lattices of metal spheres.

\section{THEORY}

We consider a square array of infinitely long metal cylinders embedded in a homogeneous medium of dielectric constant $\epsilon_{0}$; the metal of the cylinders is described by the freeelectron Drude dielectric function ${ }^{21}$

$$
\epsilon(\omega)=1-\frac{\omega_{p}^{2}}{\omega(\omega+i / \tau)},
$$

where $\tau$ represents the inelastic scattering time of bulk plasmons. In this section, we describe our theory, starting off with general considerations and going on to discuss the embedding method which we use to calculate the dispersion of the electromagnetic modes.

\section{A. General considerations}

In the long-wavelength limit, in which the radius of the cylinders is much smaller than the wavelength of the interacting electromagnetic radiation, we should be able to replace our composite material by an effective homogeneous 
medium of dielectric function $\epsilon_{\mathrm{eff}}(\omega)$, such that electromagnetic modes propagate with frequency $\omega$ given as a function of wave vector $k$ by

$$
\omega=k c / \sqrt{\epsilon_{\mathrm{eff}}(\omega)},
$$

where $c$ is the speed of light. Due to the anisotropy of the material, the effective dielectric function $\epsilon_{\text {eff }}(\omega)$ depends on the polarization of the propagating electromagnetic waves.

\section{E polarization}

In the case of electromagnetic waves with the electric field polarized along the cylinders ( $s$ polarization), we know from elementary electromagnetism ${ }^{22}$ that the electric field is continuous across the interface. This suggests that in the long-wavelength limit the composite will behave as a homogeneous medium with the dielectric function of this effective medium given by the weighted average of the dielectric functions of the constituents ${ }^{23,24}$

$$
\epsilon_{\mathrm{eff}}(\omega)=f \epsilon(\omega)+(1-f) \epsilon_{0} .
$$

Here, $f$ is the volume fraction of the cylindrical inclusions. Taking the Drude dielectric function (1) and cylinders in vacuum $\left(\epsilon_{0}=1\right)$, Eq. (3) yields

$$
\epsilon_{\mathrm{eff}}(\omega)=1-f \frac{\omega_{p}^{2}}{\omega(\omega+i / \tau)} .
$$

This shows that for this polarization the optical response of the composite material is expected to be that of free electrons in a homogeneous electron gas, but with the reduced plasma frequency $\sqrt{f} \omega_{p}$.

\section{2. $\mathrm{H}$ polarization}

For electromagnetic waves with the electric field polarized normal to the cylinders, the electric field is strongly modified by the presence of the interfaces. In the case of a two-component isotropic system composed of identical inclusions of dielectric function $\epsilon(\omega)$ in a host medium of dielectric constant $\epsilon_{0}$, the polarization $\mathbf{P}$ can be easily obtained as follows:

$$
\mathbf{P}=f \frac{\epsilon-1}{4 \pi} \mathbf{E}_{\text {in }}+(1-f) \frac{\epsilon_{0}-1}{4 \pi} \mathbf{E}_{\text {out }},
$$

where $\mathbf{E}_{\text {in }}$ and $\mathbf{E}_{\text {out }}$ represent the average electric field inside and outside the inclusions, respectively. Assuming that our composite material can be replaced by an effective homogeneous medium of dielectric function $\epsilon_{\text {eff }}(\omega)$, one can also write

$$
\mathbf{P}=\frac{\epsilon_{\mathrm{eff}}-1}{4 \pi} \mathbf{E},
$$

where $\mathbf{E}$ is the macroscopic electric field averaged over the composite:

$$
\mathbf{E}=f \mathbf{E}_{\text {in }}+(1-f) \mathbf{E}_{\text {out }} .
$$

Equations (5)-(7) yield the following relation:

$$
\left(\epsilon_{\text {eff }}-\epsilon_{0}\right) \mathbf{E}=f\left(\epsilon-\epsilon_{0}\right) \mathbf{E}_{\text {in }} .
$$

In the case of a single 2D circular inclusion embedded in an otherwise homogeneous medium, an elementary analysis ${ }^{22}$ shows that the electric field $\mathbf{E}_{\text {in }}$ in the interior of the inclusion is

$$
\mathbf{E}_{\text {in }}=\frac{u}{u-1 / 2} \mathbf{E},
$$

where

$$
u=\left[1-\epsilon / \epsilon_{0}\right]^{-1} .
$$

Introducing Eqs. (9) and (10) into Eq. (8) yields

$$
\epsilon_{\mathrm{eff}}(\omega)=\epsilon_{0}\left[1-f \frac{1}{u-1 / 2}\right] .
$$

This equation, which coincides with Eq. (27) of Ref. 13, describes for a Drude metal the surface-plasmon mode $\omega_{s}$ $=\omega_{p} / \sqrt{1+\epsilon_{0}}$ of a single cylinder.

The interaction among circular inclusions in a host medium can be introduced approximately in the framework of the Maxwell-Garnett (MG) approximation. ${ }^{25}$ The basic assumption of this approach is that the average electric field $\mathbf{E}_{\text {in }}$ within an inclusion located in a system of identical inclusions is related to the average field $\mathbf{E}_{\text {out }}$ in the medium outside as in the case of a single isolated (noninteracting) inclusion, thereby only dipole interactions being taken into account. Hence, in this approach the electric field $\mathbf{E}_{\text {in }}$ is taken to be of the form of Eq. (9) but with the macroscopic electric field $\mathbf{E}$ replaced by the electric field $\mathbf{E}_{\text {out }}$ outside, which together with Eqs. (7) and (8) yields ${ }^{24}$

$$
\epsilon_{\mathrm{eff}}(\omega)=\epsilon_{0}\left[1-f \frac{1}{u-m}\right],
$$

with $m=(1-f) / 2$. In the dilute $(f \rightarrow 0)$ limit, $m=1 / 2$ and the Maxwell-Garnett Eq. (12) yields Eq. (11).

As optical absorption by a composite is dictated by the poles of $\epsilon_{\mathrm{eff}}$, an inspection of Eq. (12) shows that for Drude cylinders in vacuum the MG approximation predicts optical absorption to occur at $\sqrt{(1-f) / 2} \omega_{p}$, which in the dilute $(f \rightarrow 0)$ limit yields the surface-plasmon frequency $\omega_{p} / \sqrt{2}$. Conversely, the energy loss of swift charged particles is dictated by the poles of the effective inverse dielectric function $\epsilon_{\text {eff }}^{-1}(\omega)$. From Eq. (12), one finds

$$
\epsilon_{\mathrm{eff}}^{-1}(\omega)=\epsilon_{0}^{-1}\left[1+f \frac{1}{u-n}\right],
$$

with $n=(1+f) / 2$. Equation (13) shows that for Drude cylinders in vacuum the MG approximation predicts the excitation of surface plasmons at $\sqrt{(1+f) / 2} \omega_{p}$, which in the dilute $(f \rightarrow 0)$ limit yields, as in the case of optical absorption, the surface-plasmon frequency $\omega_{p} / \sqrt{2}$.

\section{B. Embedding technique}

In the absence of free charges or currents, Maxwell's equations reduce to the eigenvalue equation 


$$
\boldsymbol{\nabla} \times \boldsymbol{\nabla} \times \mathbf{E}=\epsilon(\mathbf{r}) \frac{\omega^{2}}{c^{2}} \mathbf{E}
$$

where $\epsilon(\mathbf{r})$ is the spatially varying dielectric function, and we assume a magnetic permeability $\mu=1$ everywhere. To solve this equation, we use the embedding technique described in Ref. 26 and employed in Ref. 17 to investigate plasmon bands in 3D periodic arrangements of metal spheres. In this approach, the metallic cylinders are replaced by an embedding potential over their surfaces, and the electromagnetic field in between is expanded in a given basis set. With the embedding method one has, of course, complete freedom in the choice of this basis set; we use a plane-wave basis set, which represents a natural choice for periodic systems. Because the plane-wave basis is used only in the vacuum between the cylinders, convergence is very rapid.

We consider an electromagnetic wave normally incident on the structure, i.e., with the wave vector taken in the plane perpendicular to the axes of the cylinders, which we take to be the $z$ direction. With this choice, the cylinder problem simplifies considerably compared with the spheres, because the vector wave equation can be written in scalar form. In the case of $E$ polarization, the problem reduces to solving the wave equation for $E_{z}$. The other case is $H$ polarization, with the electric field perpendicular to the cylinders, in which case we have a scalar wave equation for $H_{z}$. As usual when solving Maxwell's equations, the boundary conditions on the fields are that the surface-parallel components of $\mathbf{E}$ and $\mathbf{H}$ are continuous across the surfaces of the cylinders. This is taken care of by the embedding potential.

\section{E polarization}

For $E$ polarization, Eq. (14) becomes the scalar wave equation

$$
-\nabla^{2} E_{z}=\epsilon(\mathbf{r}) \frac{\omega^{2}}{c^{2}} E_{z}
$$

In the embedding method, we derive a variational expression for the eigenvalue $\omega^{2} / c^{2}$ in terms of a trial electric field $\mathcal{E}_{z}$ defined only in the region between the cylinders, region $I$,

$$
\frac{\omega^{2}}{c^{2}}=\frac{\int_{I} d \mathbf{r} \boldsymbol{\nabla} \mathcal{E}_{z}^{*} \cdot \nabla \mathcal{E}_{z}-\int_{S} d \mathbf{r}_{S} \int_{S} d \mathbf{r}_{S}^{\prime} \mathcal{E}_{z}^{*}\left(\Sigma-\omega_{0}^{2} \frac{\partial \Sigma}{\partial \omega_{0}^{2}}\right) \mathcal{E}_{z}}{\epsilon_{0} \int_{I} d \mathbf{r} \mathcal{E}_{z}^{*} \cdot \mathcal{E}_{z}+c^{2} \int_{S} d \mathbf{r}_{S} \int_{S} d \mathbf{r}_{S}^{\prime} \mathcal{E}_{z}^{*} \frac{\partial \Sigma}{\partial \omega_{0}^{2}} \mathcal{E}_{z}} .
$$

The contribution from each cylinder is replaced by the double integral over its surface, containing the frequencydependent embedding potential $\Sigma\left(\mathbf{r}_{S}, \mathbf{r}_{S}^{\prime} ; \omega_{0}^{2} / c^{2}\right)$, evaluated at a trial frequency $\omega_{0}$. The terms involving the derivatives of the embedding potential originally arise from volume integrals through the cylinders, but provide a first-order correction so that $\Sigma$ is evaluated at the estimated frequency $\omega$ rather than the trial frequency $\omega_{0}$.
The embedding potential is defined in terms of the exact solution $E_{z}$ of the wave equation inside the cylinder, at frequency $\omega_{0}$, which matches on to the trial solution $\mathcal{E}_{z}$ over the surface of the cylinder

$$
\frac{\partial E_{z}\left(\mathbf{r}_{S}\right)}{\partial n_{S}}=\int_{S} d \mathbf{r}_{S}^{\prime} \Sigma\left(\mathbf{r}_{S}, \mathbf{r}_{S}^{\prime}\right) \mathcal{E}_{z}\left(\mathbf{r}_{S}^{\prime}\right)
$$

- it gives the exact normal derivative corresponding to the trial surface amplitude.

To solve Eq. (16), the trial function is expanded in terms of basis functions $F_{i}$,

$$
\mathcal{E}_{z}(\mathbf{r})=\sum_{i} e_{i} F_{i}(\mathbf{r}), \quad F_{i}(\mathbf{r})=\exp \left(i \mathbf{k}_{i} \cdot \mathbf{r}\right)
$$

For a 2D periodic lattice of cylinders, suitable basis functions are plane waves, where the wave vector $\mathbf{k}_{i}$ is given by $\mathbf{k}_{i}=\mathbf{k}+\mathbf{g}_{i}$, with $\mathbf{k}$ the Bloch wave vector (two dimensional in the plane perpendicular to the cylinders) and $\mathbf{g}_{i}$ is a $2 \mathrm{D}$ reciprocal lattice vector. Substituting into Eq. (16) and finding the stationary values with respect to the coefficients $e_{i}$ gives the matrix eigenvalue equation

$$
A e=\frac{\omega^{2}}{c^{2}} B e,
$$

where the $A$ and $B$ matrices are given by

$$
A_{i j}=\int_{I} d \mathbf{r} \boldsymbol{\nabla} F_{i}^{*} \cdot \nabla F_{j}-\int_{S} d \mathbf{r}_{S} \int_{S} d \mathbf{r}_{S}^{\prime} F_{i}^{*}\left(\Sigma-\omega_{0}^{2} \frac{\partial \Sigma}{\partial \omega_{0}^{2}}\right) F_{j}
$$

and

$$
B_{i j}=\epsilon_{0} \int_{I} d \mathbf{r} F_{i}^{*} \cdot F_{j}+c^{2} \int_{S} d \mathbf{r}_{S} \int_{S} d \mathbf{r}_{S}^{\prime} F_{i}^{*} \frac{\partial \Sigma}{\partial \omega_{0}^{2}} F_{j}
$$

The integrals in region $I$, over the $2 \mathrm{D}$ unit cell excluding the cylinder, are easy to evaluate,

$$
\int_{I} d \mathbf{r} \nabla F_{i}^{*} \cdot \nabla F_{j}=\left\{\begin{array}{cc}
k_{i}^{2}\left(A-\pi r^{2}\right), & i=j, \\
-2 \pi r \mathbf{k}_{i} \cdot \mathbf{k}_{j} \frac{J_{1}\left(r\left|\mathbf{k}_{i}-\mathbf{k}_{j}\right|\right)}{\left|\mathbf{k}_{i}-\mathbf{k}_{j}\right|}, & i \neq j
\end{array}\right.
$$

and

$$
\int_{I} d \mathbf{r} F_{i}^{*} \cdot F_{j}=\left\{\begin{array}{cc}
\left(A-\pi r^{2}\right), & i=j, \\
-2 \pi r \frac{J_{1}\left(r\left|\mathbf{k}_{i}-\mathbf{k}_{j}\right|\right)}{\left|\mathbf{k}_{i}-\mathbf{k}_{j}\right|}, & i \neq j
\end{array}\right.
$$

$-A$ is the area of the unit cell and $r$ is the cylinder radius.

From a multipole expansion of the solution of the scalar wave equation in cylindrical harmonics and matching onto 
the trial wave function on the surface of the cylinder (17) yields the following expression for the matrix element of the embedding potential:

$$
\begin{aligned}
\int_{S} d \mathbf{r}_{S} \int_{S} d \mathbf{r}_{S}^{\prime} F_{i}^{*} \Sigma F_{j}= & -2 \pi r \kappa \sum_{m}^{m_{\max }} \exp i m\left(\phi_{i}-\phi_{j}\right) \\
& \times \frac{J_{m}\left(k_{i} r\right) J_{m}\left(k_{j} r\right)}{J_{m}(\kappa r)} J_{m}^{\prime}(\kappa r) .
\end{aligned}
$$

Here, $J_{m}(x)$ represent Bessel functions of the first kind, ${ }^{27} \phi_{i}$ is the angle of basis wave vector $\mathbf{k}_{i}$, and $\kappa$ represents the magnitude of the (complex) wave vector of the solution of Maxwell's equations inside the cylinder at trial frequency $\omega_{0}$, $\kappa=\sqrt{\epsilon} \omega_{0} / c$. For this polarization, convergence is rapid with respect to the maximum harmonic $m_{\max }$ in the expansion.

The complex dielectric function of the lossy metal cylinders, Eq. (1), means that the frequencies of solutions of Maxwell's equations are broadened, and rather than solving the eigenvalue equation (19), we find the corresponding Green function $\Gamma\left(\mathbf{r}, \mathbf{r}^{\prime} ; \lambda\right)$ given in region $I$ by

$$
\Gamma\left(\mathbf{r}, \mathbf{r}^{\prime} ; \lambda\right)=\sum_{i j} \Gamma_{i j}(\lambda) F_{i}^{*}(\mathbf{r}) F_{j}\left(\mathbf{r}^{\prime}\right)
$$

with

$$
\sum_{k}\left(A_{i k}-\lambda B_{i k}\right) \Gamma_{k j}(\lambda)=\delta_{i j} .
$$

As we know the frequency at which the Green function is evaluated, the embedding potential is evaluated at this frequency, and the frequency-derivative terms in $A$ and $B$ cancel out in Eq. (26).

The Green function is related to the spectral density of the electric field

$$
n(\mathbf{r}, \omega)=\sum_{i} \epsilon(\mathbf{r}) E_{z, i}^{*}(\mathbf{r}) E_{z, i}(\mathbf{r}) \delta\left(\omega-\omega_{i}\right),
$$

which multiplied by $\delta \omega$ gives the electric-field intensity at point $\mathbf{r}$ in this frequency range. As the Green function can be expressed in terms of the eigenmodes and eigenfrequencies satisfying Eq. (15),

$$
\Gamma\left(\mathbf{r}, \mathbf{r}^{\prime} ; \lambda\right)=\sum_{i} \frac{E_{z, i}^{*}(\mathbf{r}) E_{z, i}\left(\mathbf{r}^{\prime}\right)}{\omega_{i}^{2} / c^{2}-\lambda},
$$

we see that the spectral density is given by

$$
n(\mathbf{r}, \omega)=\frac{2 \omega}{\pi c^{2}} \epsilon(\mathbf{r}) \operatorname{Im} \Gamma\left(\mathbf{r}, \mathbf{r} ; \omega^{2} / c^{2}+i \delta\right) .
$$

Integrating $n$ through region $I$ gives

$$
n_{I}(\omega)=\frac{2 \omega \epsilon_{0}}{\pi c^{2}} \sum_{i j} \operatorname{Im} \Gamma_{i j} \int_{I} d \mathbf{r} F_{i}^{*} \cdot F_{j}
$$

$-n_{I}$ is the quantity we calculate and plot.

\section{2. $H$ polarization}

For $H$ polarization, we solve the scalar equation for the $z$ component of the magnetic field

$$
-\nabla^{2} H_{z}=\epsilon(\mathbf{r}) \frac{\omega^{2}}{c^{2}} H_{z}
$$

and then the requirement that the surface-parallel components of $\mathbf{H}$ and $\mathbf{E}$ are continuous reduces to the continuity of $H_{z}$ and $\frac{1}{\epsilon} \partial H_{z} / \partial n_{s}$ across the surface of the cylinders. The magnetic variational principle, analogous to Eq. (16), is given by

$$
\frac{\omega^{2}}{c^{2}}=\frac{\frac{1}{\epsilon_{0}} \int_{I} d \mathbf{r} \boldsymbol{\nabla} \mathcal{H}_{z}^{*} \cdot \nabla \mathcal{H}_{z}-\int_{S} d \mathbf{r}_{S} \int_{S} d \mathbf{r}_{S}^{\prime} \mathcal{H}_{z}^{*}\left(\Sigma^{m}-\omega_{0}^{2} \frac{\partial \Sigma^{m}}{\partial \omega_{0}^{2}}\right) \mathcal{H}_{z}}{\int_{I} d \mathbf{r} \mathcal{H}_{z}^{*} \cdot \mathcal{H}_{z}+c^{2} \int_{S} d \mathbf{r}_{S} \int_{S} d \mathbf{r}_{S}^{\prime} \mathcal{H}_{z}^{*} \frac{\partial \Sigma^{m}}{\partial \omega_{0}^{2}} \mathcal{H}_{z}},
$$

where the magnetic embedding potential now satisfies

$$
\frac{1}{\epsilon_{0}} \frac{\partial H_{z}\left(\mathbf{r}_{S}\right)}{\partial n_{S}}=\int_{S} d \mathbf{r}_{S}^{\prime} \Sigma^{m}\left(\mathbf{r}_{S}, \mathbf{r}_{S}^{\prime}\right) \mathcal{H}_{z}\left(\mathbf{r}_{S}^{\prime}\right)
$$

The matrix elements for determining the eigenmodes and Green function are the same as in the case of $E$ polarization, apart from the matrix element of the embedding potential which with the definition appropriate to the magnetic case, Eq. (33), has an extra factor of $1 / \epsilon$,

$$
\begin{aligned}
\int_{S} d \mathbf{r}_{S} \int_{S} d \mathbf{r}_{S}^{\prime} F_{i}^{*} \Sigma^{m} F_{j}= & -\frac{2 \pi r \kappa}{\epsilon\left(\omega_{0}\right)} \sum_{m}^{m_{\max }} \exp i m\left(\phi_{i}-\phi_{j}\right) \\
& \times \frac{J_{m}\left(k_{i} r\right) J_{m}\left(k_{j} r\right)}{J_{m}(\kappa r)} J_{m}^{\prime}(\kappa r) .
\end{aligned}
$$

Surface-plasmon polaritons occur with $H$ polarization, and the maximum harmonic $m_{\max }$ imposes a limit to the plasmon modes over each cylinder, without which the density of states increases without limit at the planar surface-plasmon frequency $\omega_{p} / \sqrt{2}$. Controlling $m_{\max }$ enables us to study the evolution of the plasmon bands in detail. Of course there is a 
TABLE I. Convergence test for $H$-polarized electromagnetic waves in a square array or Drude cylinders in vacuum with $\bar{\omega}_{p}=1$, $\tilde{r}=1.885$. Upper table: frequencies of bands at $\tilde{k}_{x}=0.05, \tilde{k}_{y}=0$ with 121 and 241 basis functions, compared with FDTD results of Ito and Sakoda (Ref. 12). Lower table: the same for $\tilde{k}_{x}=0.5, \tilde{k}_{y}=0$.

\begin{tabular}{lccc}
\hline \hline Band & 121 & 241 & FDTD \\
\hline 1 & 0.039 & 0.039 & 0.036 \\
2 & 0.566 & 0.566 & 0.596 \\
Plasmons & $0.638-0.711$ & $0.638-0.706$ & $0.642-0.718$ \\
3 & 1.088 & 1.086 & 1.082 \\
4 & 1.161 & 1.161 & 1.164 \\
5 & 1.184 & 1.184 & 1.182 \\
1 & 0.301 & 0.301 & 0.300 \\
2 & 0.474 & 0.474 & 0.515 \\
Plasmons & $0.632-0.720$ & $0.632-0.720$ & $0.637-0.728$ \\
3 & 0.921 & 0.921 & 0.927 \\
\hline \hline
\end{tabular}

physical cutoff for the plasmons due to Landau damping, though this is much larger than the $m_{\max }$ which we use. Unlike in scattering theory, where a maximum multipole moment is also imposed, all higher values of $m$ are included in our embedding, but with a different boundary condition. In the case of $H$ polarization, the boundary condition for $m$ $>m_{\max }$ is that the surface-normal derivative of $H_{z}$ vanishes on the surface of the cylinders, corresponding to the surfaceparallel component of $\mathbf{E}$ vanishing.

Once again we evaluate the electric spectral density, as the plasmons are essentially electrostatic in nature. Using Maxwell's equations, the integrated spectral density is given by

$$
n_{I}(\omega)=\frac{2}{\pi \omega \epsilon_{0} c^{2}} \sum_{i j} \operatorname{Im} \Gamma_{i j} \int_{I} d \mathbf{r} \nabla F_{i}^{*} \cdot \nabla F_{j},
$$

where the $F_{i}$ 's are the basis functions used to expand the magnetic field between the cylinders.

\section{RESULTS AND DISCUSSION}

We have applied the embedding formalism described above to study a square array of infinitely long Drude cylinders in vacuum. We use the dimensionless reduced frequency $\widetilde{\omega}=\omega a / 2 \pi c$ and reduced wave vector $\tilde{\mathbf{k}}=\mathbf{k} a / 2 \pi$, where $a$ is the lattice constant. The reduced cylinder radius is defined as $\tilde{r}=2 \pi r / a$.

First we provide a comparison of embedding with finitedifference time-domain (FDTD) calculations by Ito and Sakoda, ${ }^{12}$ in which both space and time are discretized. They calculated the band structure for $H$ polarization for Drude cylinders in vacuum with reduced plasmon frequency $\widetilde{\omega}_{p}$ $=1$, and reduced radius $\tilde{r}=1.885$. Results for this system calculated using embedding, with 121 and 241 plane wave basis functions and $m_{\max }=6$ are shown in Table I, compared with the FDTD results, taken from Fig. 5 of Ito and Sakoda. ${ }^{12} \mathrm{We}$ see first of all that there is excellent agreement, except for

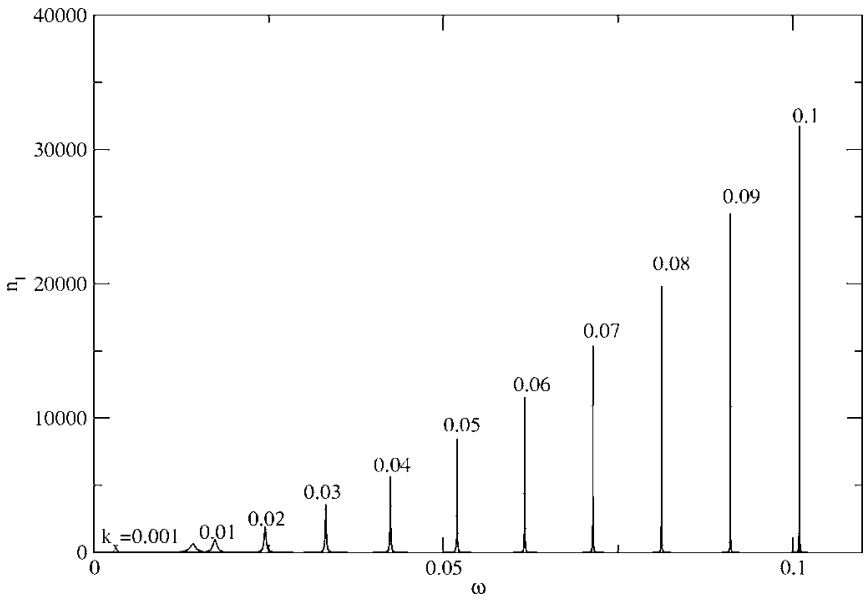

FIG. 1. Density of states $n_{I}(\omega)$ for $E$-polarized electromagnetic waves in a square array of Drude cylinders in vacuum, with $\tilde{r}$ $=0.5(f=0.02)$. Different curves correspond to increasing values of $\tilde{k}_{x}$, from 0.001 to 0.1 (with $\tilde{k}_{y}=0$ ).

band 2, where FDTD gives an energy slightly higher than our (converged) results. We also note that with only 121 plane waves the embedding method gives essentially converged results, though the individual plasmon bands within the band limits given in the tables do change slightly.

For studying the evolution of the plasmon bands, in the rest of this paper we choose the reduced plasmon frequency $\widetilde{\omega}_{p}=0.1$, which for $\mathrm{Al}\left(\omega_{p} \sim 15 \mathrm{eV}\right)$ corresponds to a lattice constant of $a \sim 83 \AA$. This value of $\widetilde{\omega}_{p}$ gives results which are universally applicable in the plasmonic regime, where the radius of the cylinders is smaller than the wavelength of the electromagnetic radiation. The reduced lifetime $\tau=2 \pi c \tau / a$ is taken as 1000, broadening peaks and enabling us to plot densities of states easily as a function of frequency.

\section{A. $E$ polarization}

For $E$-polarized electromagnetic waves (with the electric field parallel to the cylinder axis), our numerical results for the integrated density of states $n_{I}(\omega)$ and the band structure $\omega(k)$ accurately reproduce the results expected from Eqs. (1)-(3) and, therefore, the results reported in Ref. 13 for this polarization. Figure 1 gives $n_{I}(\omega)$ for various values of $k_{x}$ at a filling fraction $f=0.02 .^{28}$ The corresponding electromagnetic band structure $\omega(k)$, determined from the peak frequencies, is shown in Fig. 2 (solid circles) for three values of the filling fraction $f$. This figure shows that in the plasmonic regime under consideration the optical response of the composite material is indeed that of free electrons in a homogeneous electron gas, but with the reduced plasma frequency $\sqrt{f} \omega_{p}$ (solid lines in Fig. 2) expected from Eqs. (1)-(3).

The 2D periodicity of the composite material introduces frequency gaps in the band structure, but these are remarkably small. For cylinders with a reduced radius $\tilde{r}=2$, corresponding to $f=0.32$, the first band gap at the center of the side of the 2D Brillouin zone is 0.0035 in reduced units, and for touching cylinders with $f=0.79$ it is 0.0028 .

\section{B. $H$ polarization}

For $H$-polarized electromagnetic waves (with the electric field normal to the axes of the cylinders), our results are 


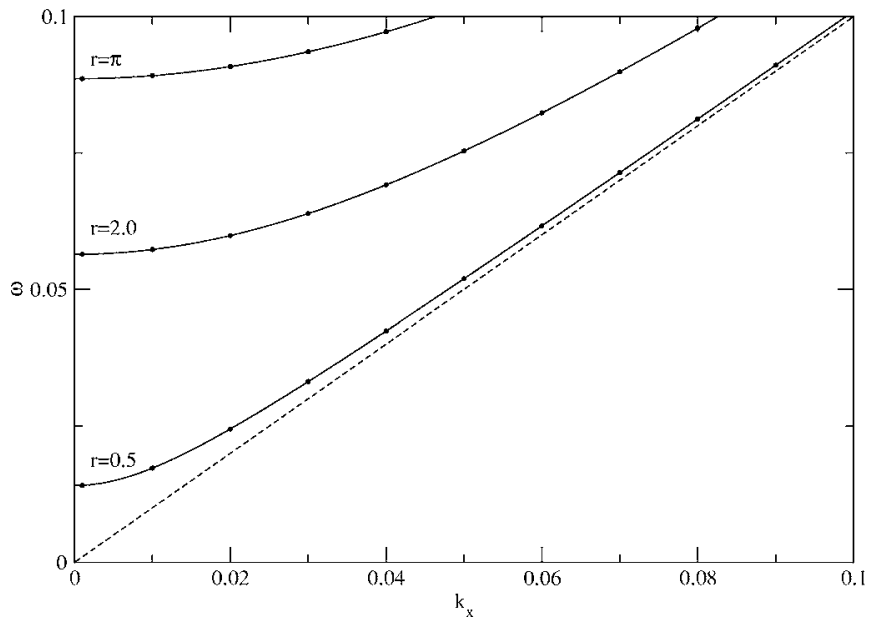

FIG. 2. E-polarization band structure as a function of $\tilde{k}_{x}$ (with $\tilde{k}_{y}=0$ ), for cylinders of radius $\tilde{r}=0.5,2.0$, and $\pi$ (touching cylinders); the corresponding filling fractions $f$ are $0.02,0.32$, and 0.79 , respectively. The solid circles represent the band structure that we have obtained from the peaks in our calculated density of states $n_{I}(\omega)$. The solid lines correspond to a homogeneous electron gas with the reduced plasma frequency $\sqrt{f} \omega_{p}$. The dashed line is the light line, $\omega=c k$.

rather similar to those found for a 3D lattice of metal spheres, ${ }^{17}$ with almost dispersionless surface plasmons on the cylinders and the light line interacting with one of the two dipole plasmons. A significant difference is that in the case of an isolated Drude metallic sphere, the dispersionless Mie plasmons have frequencies given by

$$
\omega_{l}=\omega_{p} \sqrt{\frac{l}{2 l+1}},
$$

where $l$ is the multipole quantum number, whereas for an isolated Drude cylinder all the surface plasmons have the frequency $\omega_{p} / \sqrt{2}$, irrespective of quantum number $m$. This holds for lattices with low filling fraction $(f \rightarrow 0)$, and consequently low interaction between the plasmon modes, resulting in a narrower range of plasmon bands in the case of cylinders. For larger filling fractions, the interactions between the cylinders dominate, and produce a wider range of bands, similar to the spherical case.

\section{Density of states}

We start off by considering the integrated density of states. Figure 3 shows $n_{I}(\omega)$ at three wave vectors, for cylinders with $\tilde{r}=1$ corresponding to $f=0.08$. The cylindrical surface plasmons constitute the dispersionless peaks near $\widetilde{\omega}_{p} / \sqrt{2}=0.071$, with the light line dispersing through the plasmons and interacting with a dipole mode.

In order to study the plasmons in more detail, we plot in Fig. 4 the plasmon peaks for cylinders with $\tilde{r}=1$ (as in Fig. 3 ) but now at a small wave vector $\left(\tilde{k}_{x}=0.001\right)$ and various values of $m_{\max }: 1,2,3$, and 4 . We see that at this rather small filling fraction $(f=0.08)$, the plasmon structure is very narrow-much narrower than in the spherical case. ${ }^{17}$ The

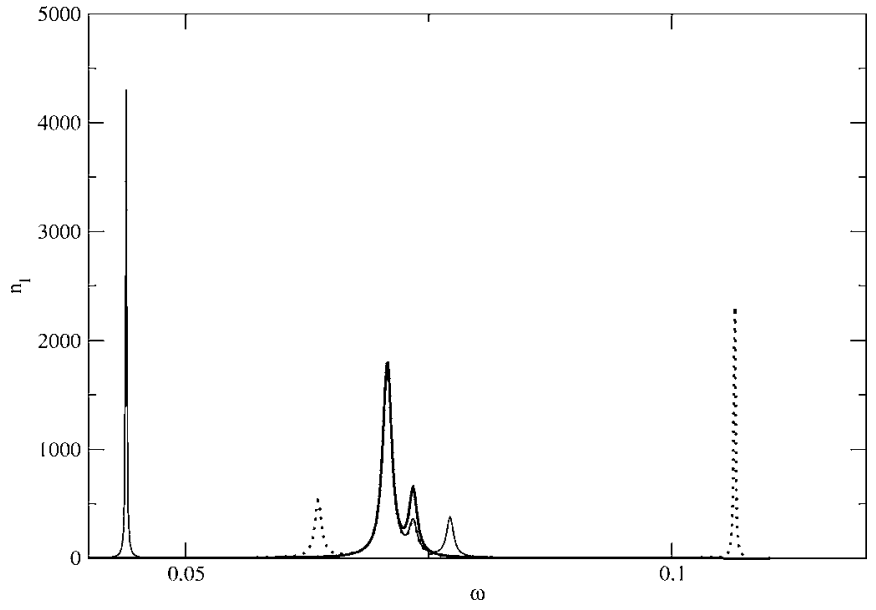

FIG. 3. Density of states $n_{I}(\omega)$ for $H$-polarized electromagnetic waves in a square array of Drude cylinders in vacuum, with $\tilde{r}$ $=1.0(f=0.08), m_{\max }=4, \tilde{k}_{y}=0$, and varying $\tilde{k}_{x}: 0.001$ (thick solid line), 0.05 (thin solid line), and 0.1 (dotted line).

effect of increasing $m_{\max }$ is simply to increase the height of the peak at $\widetilde{\omega}=0.071$, or $\widetilde{\omega}_{p} / \sqrt{2}$. In this sense, the results do not converge as $m_{\max }$ increases. But this is physically correct-the plasmon peak at $\widetilde{\omega}_{p} / \sqrt{2}$ must increase without limit as the number of allowed modes increases. However, the rest of the structure clearly converges, as we see from Fig. 4.

This behavior is confirmed for larger cylinder radii, when the electrostatic interaction between the cylinders broadens the plasmon structure. Figure 5 shows the density of states at a wave vector close to zero $(k=0.001$, as in Fig. 4), for a cylinder of radius $\tilde{r}=2.62$ corresponding to a packing fraction of $f=0.55$. We see that the wings of the plasmon structure still converge with $m_{\max }$, and once again increasing $m_{\max }$ increases the height of the central peak. For touching cylinders (Fig. 6), the density of states broadens to give plasmon structure between a rather low frequency and the bulk plas-

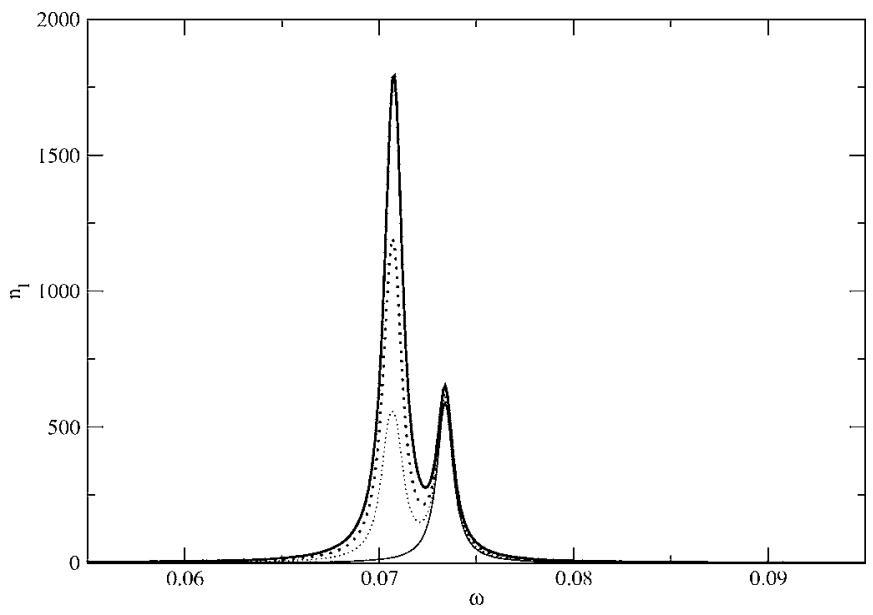

FIG. 4. Density of states $n_{I}(\omega)$ for $H$-polarized electromagnetic waves in a square array of Drude cylinders in vacuum, with $\widetilde{r}$ $=1.0(f=0.08)$, wave vector $\tilde{\mathbf{k}}=(0.001,0)$, and varying $m_{\max }: 1$ (thin solid line), 2 (stippled line), 3 (dotted line), and 4 (thick solid line). 


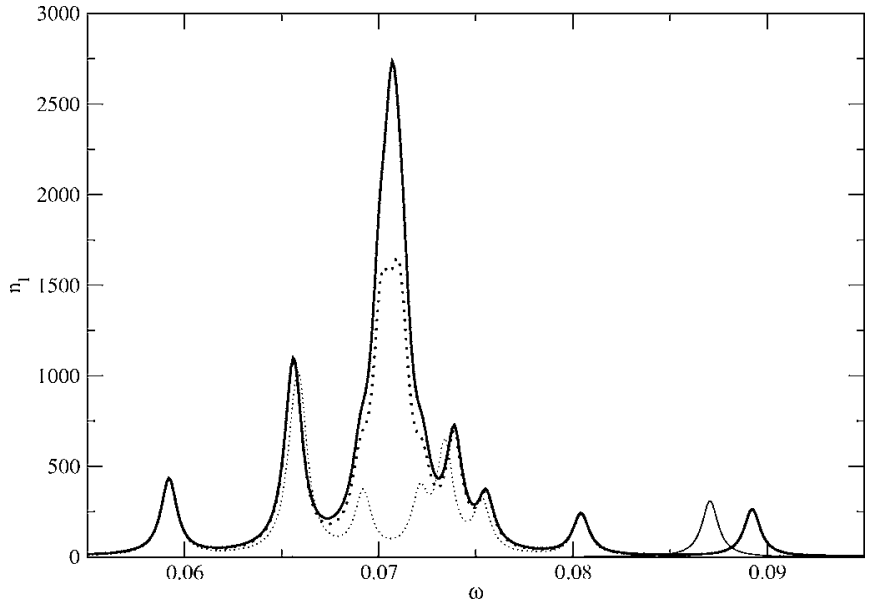

FIG. 5. Density of states $n_{I}(\omega)$ for $H$-polarized electromagnetic waves in a square array of Drude cylinders in vacuum, with $\tilde{r}$ $=2.62(f=0.55)$, wave vector $\tilde{\mathbf{k}}=(0.001,0)$, and varying $m_{\max }: 1$ (thin solid line), 6 (stippled line), 10 (dotted line), and 12 (thick solid line).

mon frequency $\widetilde{\omega}_{p}=0.1$. In this case, we cannot distinguish a central peak at the surface plasmon frequency, and increasing $m_{\max }$ alters the structure within the entire plasmon rangethough the plasmon limits converge. This is similar to the behavior we found earlier for touching spheres. ${ }^{17}$

The size of basis set we need for the convergence of these calculations is rather small, about 350 basis functions for the highest $m_{\max }$ under consideration: $m_{\max }=4$ in Figs. 3 and 4 and $m_{\max }=12$ in Figs. 5 and 6.

\section{Photonic band structure}

The peaks in the integrated density of states $n_{I}(\omega)$ correspond to normal modes of the system, and plotting the frequencies as a function of wave vector we obtain the photonic band structures shown in Figs. 7-9. Also plotted in these

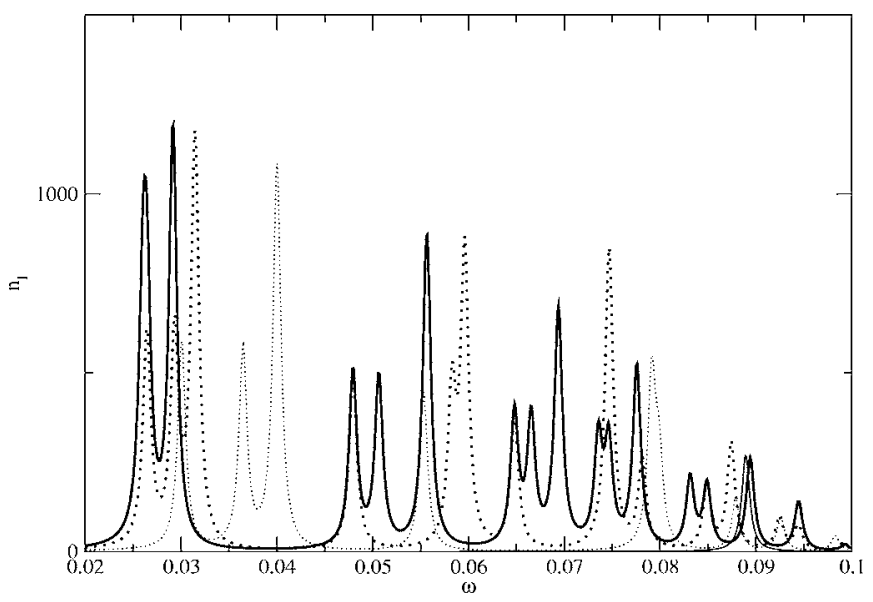

FIG. 6. Density of states $n_{I}(\omega)$ for $H$-polarized electromagnetic waves in a square array of touching Drude cylinders in vacuum, with $\tilde{r}=\pi(f=0.79)$, wave vector $\tilde{\mathbf{k}}=(0.001,0)$, and varying $m_{\max }: 1$ (thin solid line), 6 (stippled line), 10 (dotted line), and 12 (thick solid line).

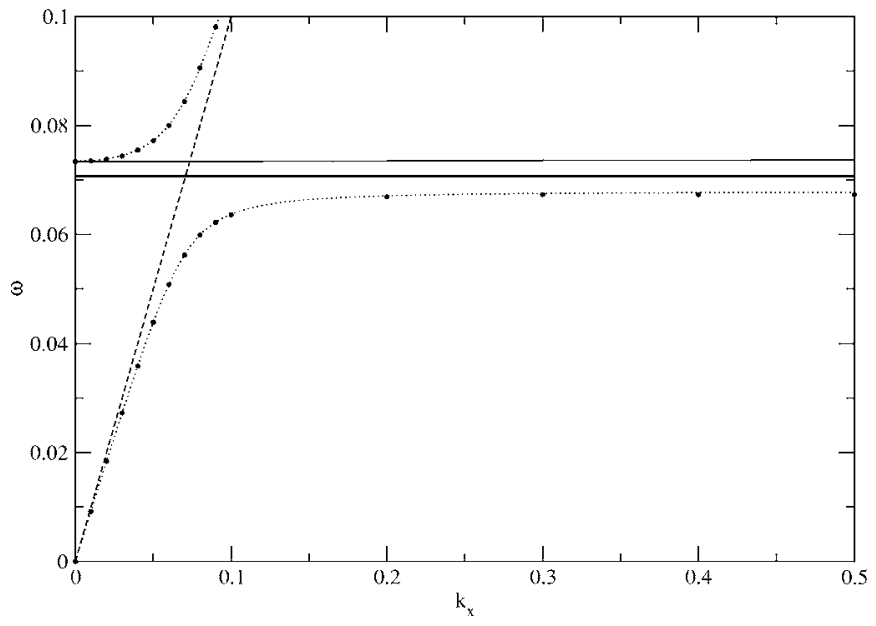

FIG. 7. $H$-polarization band structure as a function of $\tilde{k}_{x}$ (with $\tilde{k}_{y}=0$, i.e., along the $\bar{\Gamma} \bar{M}$ direction), for cylinders of radius $\tilde{r}=1.0$ $(f=0.08)$. The solid lines and circles represent the band structure (converged in $m_{\max }$ ) that we have obtained from the peaks in our calculated density of states $n_{I}(\omega)$. The dotted lines represent the $\omega(k)$ curves derived from Eq. (2) with the MG effective dielectric function $\epsilon_{\mathrm{eff}}(\omega)$ of Eq. (12).

figures (dotted lines) are the $\omega(k)$ curves derived from Eq. (2) with the MG effective dielectric function $\epsilon_{\text {eff }}(\omega)$ of Eq. (12). The limit of the lower branch occurs at $\sqrt{(1-f) / 2} \omega_{p}$, where the MG $\epsilon_{\text {eff }}(\omega)$ has a pole, and the starting frequency of the upper branch occurs at $\sqrt{(1+f) / 2} \omega_{p}$, where the MG $\epsilon_{\text {eff }}^{-1}(\omega)$ has a pole. ${ }^{29}$ In the dilute $(f \rightarrow 0)$ limit, both the limit of the lower branch and the starting frequency of the upper branch occur at the planar surface-plasmon frequency $\omega_{p} / \sqrt{2}$.

The band structure in Fig. 7 is for a small cylinder radius $\widetilde{r}=1$ (corresponding to a filling fraction of $f=0.08$ ), and exhibits an infinite number of dispersionless plasmon modes at the planar surface-plasmon frequency $\widetilde{\omega}_{p} / \sqrt{2}$. Light (singly degenerate, as we are only dealing with $H$ polarization) interacts with one of the dipole plasmons to give the light line

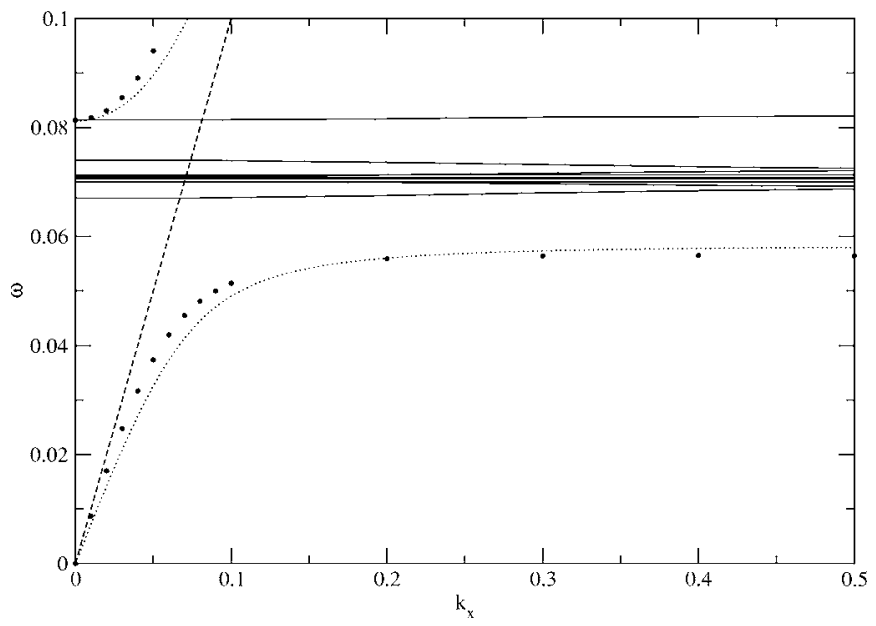

FIG. 8. As in Fig. 7, but now for cylinders of radius $\tilde{r}=2.0(f$ $=0.32$ ). This is converged in $m_{\max }$. 


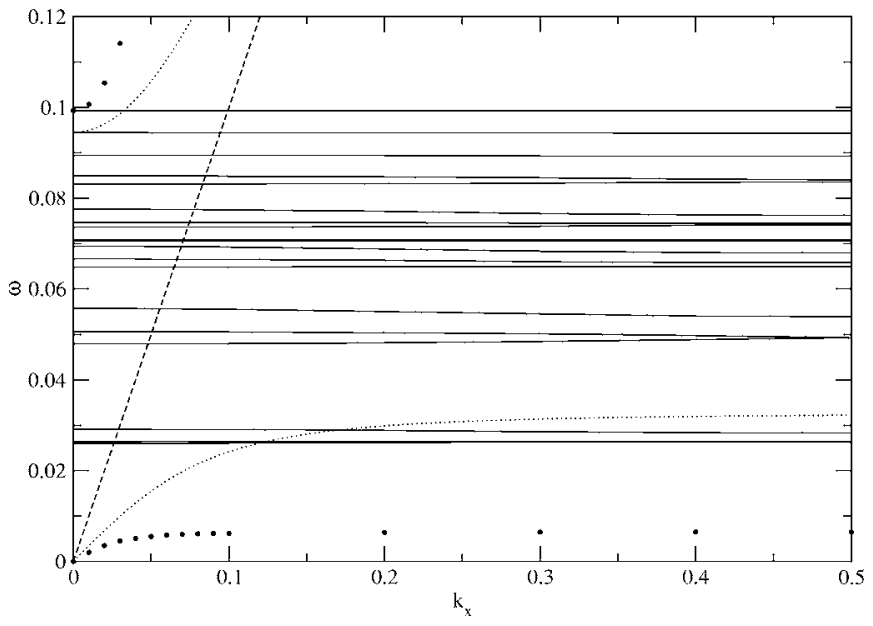

FIG. 9. As in Fig. 7, but now for touching cylinders of radius $\tilde{r}=\pi(f=0.79) . m_{\max }=12$, and although the band edges have converged, the individual bands have not.

(represented by solid circles), which is reproduced almost exactly by MG theory ${ }^{30}$ (dashed lines). The second dipole plasmon gives the almost dispersionless band joining the upper branch of the light line at zero wave vector. The dipoleactive light line branches span the multipole plasmon branches here, all at $\widetilde{\omega}_{p} / \sqrt{2}$, quite different from the case of a lattice of metal spheres, where it is only for larger filling fractions that the light branches enclose the multipole plasmons.

At the larger filling fraction $f=0.32$ considered in Fig. 8, the dipole modes and the light branches move further away from the planar surface-plasmon frequency, again closely following the MG curves. The multipole plasmon modes of lowest order are slightly broadened around $\widetilde{\omega}_{p} / \sqrt{2}$, but again there is remarkably little dispersion. There is a considerable difference between these results and those for the lattice of spheres with the same radius, which shows a much wider spread of multipole frequencies, and much more dispersion.

With touching cylinders (Fig. 9), all multipole bands spread out and MG breaks down badly. The frequency range of the multipole plasmon bands is not quite as wide as in the case of touching spheres, and the lower band edge seems to converge at $\widetilde{\omega} \approx 0.026$ with $m_{\max }=12$. (In the case of spheres, the lower band edge at $\widetilde{\omega} \approx 0.02$ was still dropping at a maximum multipole value of 12 .)

\section{CONCLUSIONS}

We have shown that as in the case of a lattice of metallic spheres ${ }^{17,26}$ the embedding method provides a very economical method of calculating electromagnetic waves in a lattice of metallic cylinders, ${ }^{31}$ which unlike other methods allows to describe accurately plasmon modes in the whole range of filling fractions from the dilute limit to the case of touching wires. For the case of $H$ polarization, where surface plasmons dominate the normal modes, the results are quite similar to those obtained for a lattice of metallic spheres, except that the frequency range of the multipole modes is narrower. For $E$ polarization, the system behaves almost exactly like a dilute electron gas, with a reduced plasmon frequency and only minute band gaps at the Brillouin zone boundaries.

The method and results presented here are for the case of the wave vector lying in the plane perpendicular to the cylinders. We are also considering the general case of arbitrary wave vector, and this will be the subject of a subsequent paper.

\section{ACKNOWLEDGMENTS}

J.M.P. acknowledges partial support by the University of the Basque Country, the Basque Unibertsitate eta Ikerketa Saila, the Spanish Ministerio de Educación y Ciencia, and the EC 6th framework Network of Excellence NANOQUANTA (Grant No. NMP4-CT-2004-500198).
${ }^{1}$ J. B. Pendry, Science 285, 1687 (1999).

${ }^{2}$ W. L. Barnes, A. Dereux, and T. W. Ebbesen, Nature (London) 424, 824 (2003).

${ }^{3}$ E. Prodan, C. Radloff, N. J. Halas, and P. Nordlander, Science 302, 419 (2003).

${ }^{4}$ P. Andrew and W. L. Barnes, Science 306, 1002 (2004).

${ }^{5}$ S. A. Maier and H. A. Atwater, J. Appl. Phys. 98, 011101 (2005).

${ }^{6}$ E. Ozbay, Science 311, 189 (2006).

${ }^{7}$ H. Raether, Surface Plasmons on Smooth and Rough Surfaces and on Gratings, Vol. 111 of Springer Tracks in Modern Physics (Springer, New York, 1988).

${ }^{8}$ J. M. Pitarke, V. M. Silkin, E. V. Chulkov, and J. M. Pitarke, Rep. Prog. Phys. 70, 1 (2007).

${ }^{9}$ R. English, J. M. Pitarke, and J. B. Pendry, Surf. Sci. 454, 1090 (2000).

${ }^{10}$ V. Kuzmiak, A. A. Maradudin, and F. Pincemin, Phys. Rev. B 50, 16835 (1994)

${ }^{11}$ H. van der Lem, J. Opt. A, Pure Appl. Opt. 2, 395 (2000).
${ }^{12}$ T. Ito and K. Sakoda, Phys. Rev. B 64, 045117 (2001).

${ }^{13}$ A. L. Pokrovsky and A. L. Efros, Phys. Rev. B 65, 045110 (2002).

${ }^{14}$ J. B. Pendry, J. Mod. Opt. 41, 209 (1994).

${ }^{15}$ A. Moroz, Phys. Rev. Lett. 83, 5274 (1999).

${ }^{16}$ V. Yannopapas, A. Modinos, and N. Stefanou, Phys. Rev. B 60, 5359 (1999).

${ }^{17}$ R. Kemp and J. E. Inglesfield, Phys. Rev. B 65, 115103 (2002).

${ }^{18}$ J. M. Pitarke, F. J. García-Vidal, and J. B. Pendry, Phys. Rev. B 57, 15261 (1998).

${ }^{19}$ J. M. Pitarke, F. J. Garcia-Vidal, and J. B. Pendry, Surf. Sci. 433, 605 (1999).

${ }^{20}$ S. Riikonen, I. Romero, and F. J. García de Abajo, Phys. Rev. B 71, 235104 (2005).

${ }^{21}$ N. W. Ashcroft and N. D. Mermin, Solid State Physics (Saunders, Philadelphia, 1976).

${ }^{22}$ J. D. Jackson, Classical Electrodynamics (Wiley, New York, 1980). 
${ }^{23}$ D. J. Bergman, Phys. Rep., Phys. Lett. 43, 378 (1978); D. J. Bergman and D. Stroud, Solid State Phys. 46, 147 (1992).

${ }^{24}$ The effective dielectric tensor of a lattice of metallic cylinders was reported in Ref. 13, at all wave vectors of the electromagnetic waves, in the dilute $(f \rightarrow 0)$ limit. When the wave vector is perpendicular to the axis of the cylinders, their Eq. (23) for $s$ polarization yields our Eq. (3).

${ }^{25}$ See, e.g., C. F. Bohren and D. R. Huffman, Absorption and Scattering of Light by Small Particles (Wiley, New York, 1983).

${ }^{26}$ J. E. Inglesfield, J. Phys. A 31, 8495 (1998); R. Kemp and J. E. Inglesfield, Phys. Rev. B 65, 115103 (2002).

${ }^{27}$ M. Abramowitz and I. A. Stegun, Handbook of Mathematical Functions (Dover, New York, 1964).
${ }^{28}$ For a 2D square array of cylinders of radius $r$, and lattice constant $a$, the filling fraction is $f=\widetilde{r}^{2} / 4 \pi, \widetilde{r}$, being the reduced radius: $\widetilde{r}=2 \pi r / a$.

${ }^{29}$ This is in contrast with the less accurate results reported in Ref. 13 for $p$ polarization, obtained from Eq. (11) [their Eq. (27)], where the limit of the lower branch and the starting frequency of the upper branch occur at $\omega_{p} / \sqrt{2}$ and $(1+f) \omega_{p} / \sqrt{2}$, respectively. ${ }^{30}$ In the dilute $(f \rightarrow 0)$ limit, all plasmon modes would accumulate at the planar surface-plasmon frequency $\widetilde{\omega}_{p} / \sqrt{2}$, and the splitting of the light line would be negligible.

${ }^{31}$ Calculations of band structures of a lattice of spheres were compared in Ref. 26 with the results obtained with other plane-wave methods, showing that embedding needed far fewer plane waves, as expected. 